\title{
CÂNCER DE MAMA: \\ estudo retrospectivo em um município do sul do Brasil
}

\author{
BREAST CANCER: \\ A retrospective study in the municipality of southern Brazil
}

\author{
Hedioneia Maria Foletto Pivetta', Adriana Cielo², Marina Segala², \\ Gustavo do Nascimento Petter ${ }^{2}$, Thais Nogueira de Oliveira Martins ${ }^{3}$, Betina Pivetta Vizzotto ${ }^{4}$, \\ Elenir Terezinha Rizzetti Anversa ${ }^{5}$, Melissa Medeiros Braz ${ }^{6}$
}

\section{RESUMO}

O objetivo deste estudo foi identificar características sociodemográficas, reprodutivas, de diagnóstico e tratamento de mulheres com câncer de mama em Santa Maria, RS. Estudo quantitativo transversal retrospectivo de 2008 a 2012, realizado por análise em prontuários de mulheres com câncer de mama residentes no Município estudado. Os dados foram analisados através do Software SPSS 10.0. Análises exploratórias univariadas das variáveis primárias e secundárias e estatística descritiva foram realizadas. Foram analisados 273 prontuários. As mulheres caracterizaram-se como da cor branca, $59,6 \%$ casadas, com média de idade no momento do diagnóstico de 56,1 anos, idade média da menarca 13 anos, da menopausa 47,23 anos e da primeira gestação 22,73 anos. Mastectomia radical e hormonioterapia foram os tratamentos mais prevalentes. A rede pública constituiu-se em porta de entrada para diagnóstico e tratamento no Município estudado. Menarca precoce e menopausa tardia não se destacaram como risco para desenvolvimento da doença nesta amostra.

Descritores: Neoplasia da Mama; Mulheres; Serviços de Saúde

\section{ABSTRACT}

The objective of this study was to identify sociodemographic, reproductive, diagnosis and treatment of women with breast cancer in Santa Maria, RS. Retrospective cross-sectional quantitative study 2008-2012 conducted by analysis of medical records of women with breast cancer living in the studied municipality. Data were analyzed using SPSS 10.0 software. Univariate exploratory analyzes of primary and secondary variables, and descriptive statistics were performed. 273 records were analyzed. Women are characterized as white color, $59,6 \%$ marrieds, with a mean age at diagnosis of 56.1 years, mean age at menarche 13 years, 47.23 years of menopause and first pregnancy 22.73 years. Radical mastectomy and hormone therapy were the most prevalent treatments. The network public constituted an entry point for diagnosis and treatment in the studied municipality. Early menarche and late menopause did not stand out as a risk for developing the disease in this sample.
${ }^{1}$ Doutora em Educação pela Universidade Federal de Santa Maria (UFSM), Santa Maria, RS, Brasil.

${ }^{2}$ Graduada em Fisioterapia pela Universidade Federal de Santa Maria (UFSM),Santa Maria, RS, Brasil.

${ }^{3}$ Graduanda em Fisioterapia na Universidade Federal de Santa Maria (UFSM), Santa Maria, RS, Brasil.

${ }^{4}$ Graduada em Fisioterapia pelo Centro Universitário Franciscano, (UNIFRA), Santa Maria, RS, Brasil.

${ }^{5}$ Mestre em Epidemiologia pela Universidade Federal do Rio Grande do Sul (UFRGS), Porto Alegre, RS, Brasil.

${ }^{6}$ Doutora em Engenharia de Produção pela Universidade Federal de Santa Catarina (UFSC), Florianópolis, SC, Brasil. 


\section{Introdução}

Nas últimas décadas, a incidência do câncer de mama vem crescendo, transformando-se em um grave problema de saúde pública mundial ${ }^{1}$ responsável por $20,8 \%$ dos novos casos de câncer a cada ano no Brasil2 ${ }^{2}$, e pelos maiores índices de mortalidade no mundo, principalmente na população feminina. Conforme a estimativa para 2014, do Instituto Nacional de Câncer, só no Rio Grande do Sul (RS) foi de 5.030 novos casos².

$O$ câncer de mama pertence a um grupo heterogêneo de doenças, com comportamentos distintos, que podem ser observados pelas diferentes assinaturas genéticas e diferentes respostas clínicas ${ }^{3,4}$. Acomete frequentemente mulheres em uma faixa etária acima de 40 anos, entretanto, mundialmente tem aumentado significativamente a sua incidência em faixas etárias mais jovens ${ }^{5,6}$.

Diante da elevada incidência do câncer de mama e, considerando um melhor prognóstico quando a doença é detectada precocemente, considera-se importante a instituição de ações de rastreamento para que o diagnóstico seja realizado em estádios iniciais, o que melhora as possibilidades de intervenções conservadoras e com melhor prognóstico de sobrevida ${ }^{7}$. As ações de controle do câncer de mama exigem uma rede de saúde que contemple todos os níveis de atenção, sendo empregadas tecnologias com diferentes densidades. Em 2011 foram traçadas estratégias para o controle do câncer de mama no Brasil com o lançamento do Plano de Fortalecimento da Rede de Prevenção, Diagnóstico e Tratamento do Câncer ${ }^{8}$.

Entretanto, para o planejamento e implementação de medidas para o diagnóstico precoce do câncer de mama, bem como de tratamento eficaz, é necessário primeiramente reconhecer o cenário local identificando as características das mulheres que já desenvolveram a doença.

Desse modo, diante dos elevados indicadores de saúde que apontam o aumento de novos casos de câncer de mama no Brasil, especialmente na região sul e, tendo em vista as taxas de morbimortalidade causadas pela doença, justifica-se o desenvolvimento da pesquisa, que através de estudo retrospectivo secundário, teve como objetivo identificar características sociodemográficas, reprodutivas, de diagnóstico e tratamento de mulheres com câncer de mama no município de Santa Maria, RS.

\section{Metodologia}

Foi realizado um estudo transversal quantitativo de caráter retrospectivo do período de 2008 a 2012, utilizando dados secundários dos prontuários de mulheres com câncer de mama residentes em Santa Maria, RS. O estudo foi aprovado pelo Comitê de Ética em Pesquisa da Universidade Federal de Santa Maria - UFSM conforme parecer número 370.708/2013.

Os dados foram coletados no ambulatório de saúde da mulher, serviço municipal de mastologia, referência para as Unidades Básicas de Saúde (UBS) do Município e do arquivo de prontuários do Hospital Universitário de Santa Maria (HUSM) no ano de 2013. Estes locais consistem em porta de entrada para o Sistema Único de Saúde (SUS), do município de Santa Maria para o diagnóstico e tratamento do câncer de mama.

Para o levantamento de dados foi elaborado pelos pesquisadores um questionário composto por questões abertas e fechadas, que contemplam as características sociodemográficas, reprodutivas, de diagnóstico e tratamento para o câncer de mama. Quanto aos aspectos sociodemográficos, buscaram-se dados quanto a idade, cor, profissão, escolaridade e estado civil. As características reprodutivas levantadas foram a idade da menarca, da menopausa e da primeira gestação, assim como tempo de aleitamento materno. Contemplou-se ainda aspectos relativos ao diagnóstico, como local de primeira suspeita/diagnóstico e classificação BIRADS, e de tratamento, cirurgia de maior prevalência e seguimento. Pacientes que apresentaram prontuários nos dois locais de coleta, tiveram as informações tabuladas do registro mais completo.

Foram incluídos prontuários de todas as mulheres com diagnóstico de câncer de mama confirmado e residentes no município de Santa Maria, RS. Excluiu-se do estudo prontuários médicos com diagnóstico inconclusivo para câncer de mama.

Primeiramente os dados foram tabulados através do Software Microsoft Excel, versão 2007. A tabulação foi realizada diariamente, bem como foram realizadas análises de monitoramento durante este processo. Após o término da coleta e tabulação dos dados, iniciou-se o processo de análise estatística pelo Software SPSS 10.0 (Statistical Package for the Social Sciences Inc., Chicago, Estados Unidos). Análises exploratórias univariadas das variáveis primárias e secundárias, bem como a estatística descritiva foram realizadas. Os dados serão apresentados na forma de tabelas. 


\section{Resultados e Discussão}

Os dados apresentados a seguir são referentes aos prontuários que continham as informações específicas para as variáveis analisadas, sendo os percentuais apresentados relativos a estes prontuários. Isso equivale a dizer que nem todos os prontuários analisados apresentaram os dados da investigação. Em relação aos locais de coleta de dados foram analisados 148 prontuários no Serviço Municipal de Mastologia e 125 no Hospital Universitário de Santa Maria, totalizando uma amostra de 273 prontuários.

A tabela 1 apresenta o percentual relativo ao local de primeira suspeita ou diagnóstico do câncer de mama no município, sistematizados por nível de atenção à saúde no sistema público, assim como no setor privado. 0 local com maior número de primeira suspeita/diagnóstico foi a rede pública de atenção primária, com $40 \%$.

Tabela 1 - Local de primeira suspeita ou diagnóstico em relação aos níveis de atenção à saúde.

\begin{tabular}{c|c|c}
\hline Local de primeira suspeita ou diagnóstico & N & \% Relativo \\
\hline Atenção Primária SUS* & 85 & 40 \\
\hline Atenção Secundária SUS** & 34 & 16 \\
\hline Atenção Terciária SUS** & 57 & 27 \\
\hline Rede privada & 36 & 17 \\
\hline
\end{tabular}

*Atenção primária do SUS no município: Unidades Básicas de Saúde e ESF

${ }^{*}$ Atenção secundária do SUS no município: Unidade de Saúde $\mathrm{N}^{a} \mathrm{Sr}{ }^{\mathrm{a}}$ do Rosário/Ambulatório de saúde da mulher

***Atenção terciária do SUS no município: Hospital Universitário de Santa Maria

Dados sociodemográficos, escolaridade e profissão, não foram encontrados nos prontuários. A cor branca foi a mais prevalente nos prontuários com percentual relativo de $91,7 \%$, seguido de preta $(6,4 \%)$ e parda $(1,8 \%)$. Quanto à situação conjugal $59,6 \%$ estavam registradas como casadas, $15,4 \%$ viúvas, $14,4 \%$ solteiras e $10,6 \%$ separadas.

Quanto à idade no momento do diagnóstico identificou-seque a mulher mais jovem tinha 21 anos e a mais senil 86 anos, enquanto a média de idade foi de 56,1 $\pm 12,91$. O número de casos por faixa etária está apresentado na Tabela 2. Os valores referentes às características das mulheres com câncer de mama, relacionados com a vida reprodutiva da mulher estão na Tabela 3. Os dados referentes ao tempo de aleitamento materno não foram encontrados nos prontuários.

Tabela 2 - Número de diagnósticos de câncer de mama por faixa etária

\begin{tabular}{c|c|c}
\hline Idade do diagnóstico & $\mathbf{N}$ & \% Relativo \\
\hline $21-30$ & 04 & 1,48 \\
\hline $31-40$ & 19 & 7,03 \\
\hline $41-50$ & 84 & 31,11 \\
\hline $51-60$ & 70 & 25,92 \\
\hline $61-70$ & 55 & 20,37 \\
\hline $71-80$ & 28 & 10,37 \\
\hline $81-90$ & 10 & 3,70 \\
\hline
\end{tabular}

Tabela 3 - Características reprodutivas apresentadas em média de idade

\begin{tabular}{c|c}
\hline Fatores de risco & Idade (Média $\pm \mathbf{D P}$ ) \\
\hline Menarca & $13 \pm 1,8$ \\
\hline Menopausa & $47,23 \pm 7,65$ \\
\hline Primeira gestação & $22,73 \pm 5,65$ \\
\hline
\end{tabular}


As características de diagnóstico encontradas foram quanto aos achados mamográficos, sendo o mais prevalente o Birads5 (58\%) e o Birads4 (31\%). O Birads (Breast Image Reporting and Data System) é um sistema padronizado utilizado internacionalmente para uniformizar os relatos de radiologia quando se analisam as imagem de mamografia de rastreamento, classificado de 0 a 6 , sendo 0 quando imagens adicionais são necessárias e 6 para achados mamográficos já biopsiados cujo diagnóstico anátomo-patológico é de câncer de mama, antes da terapia definitiva.

Os dados quanto ao tamanho do tumor e estádio não foram investigados no momento da coleta. $O$ tipo de cirurgia que a maioria das mulheres do estudo foi submetida foi a mastectomia radical $(66 \%)$, seguido da quadrantectomia com linfadenectomia axilar (29\%).

O seguimento da maioria dos casos, no momento da coleta de dados,foi a hormonioterapia (41\%) e em fase de monitoramento por consulta de rotina (34\%). A tabela 4 apresenta as variáveis relativas ao diagnóstico mamográfico do câncer, o tipo de cirurgia prevalente e o seguimento do câncer de mama.

Tabela 4 - Variáveis de diagnóstico e tratamento do câncer de mama

\begin{tabular}{|c|c|c|}
\hline Diagnóstico e tratamento & $\mathbf{n}$ & \% Relativo \\
\hline \multicolumn{3}{|l|}{ Diagnóstico mamográfico } \\
\hline BiradsO & 0 & 0 \\
\hline Birads 1 & 1 & 2 \\
\hline Birads2 & 3 & 6 \\
\hline Birads3 & 0 & 0 \\
\hline Birads4 & 15 & 31 \\
\hline Birads5 & 28 & 58 \\
\hline Birads6 & 1 & 2 \\
\hline \multicolumn{3}{|l|}{ Tipo de cirurgia } \\
\hline Mastectomia radical & 139 & 66 \\
\hline Quadrantectomia+ linfadenectomia axilar & 62 & 29 \\
\hline Outros & 10 & 5 \\
\hline \multicolumn{3}{|l|}{ Seguimento } \\
\hline Hormonioterapia & 84 & 41 \\
\hline Tratamento concluído & 11 & 5 \\
\hline Em Radioterapia & 8 & 4 \\
\hline Em Quimioterapia & 20 & 10 \\
\hline Em consulta de rotina & 70 & 34 \\
\hline Óbito & 12 & 6 \\
\hline
\end{tabular}

O câncer de mama constitui o tipo de câncer mais incidente em mulheres e, embora tem-se observado que esta doença aumenta proporcionalmente com a idade, mulheres mais jovens tem desenvolvido a doença. Entretanto, a redução da morbimortalidade associada a esta patologia está diretamente relacionada com a detecção precoce e ao tratamento efetivo, embora $60 \%$ dos tumores de mama no Brasil ainda sejam diagnosticados em estádios avançados 9

Este estudo teve por objetivo identificar características sociodemográficas, reprodutivas, de diagnóstico e tratamento de mulheres com câncer de mama em Santa Maria, RS. A pesquisa apresenta como dado relevante a constatação de que, do total da amostra estudada, $83 \%$ das mulheres teve a rede de saúde pública como local de primeira suspeita ou diagnóstico para o câncer de mama. Deste montante, $40 \%$ representa a atenção primária de saúde. Cabe destacar que, mediante os dados encontrados, não é possível inferir se a atenção básica, assim como os outros níveis de atenção, é responsável pela suspeita ou pelo diagnóstico definitivo, uma vez que os dados dos prontuários não permitem essa afirmação sendo, por este motivo foi assim sistematizado. 
É provável que a atenção básica tenha sido o local de primeira suspeita e que o diagnóstico definitivo tenha se efetivado nos serviços de referência de nível secundário ou terciário, pois assim se efetiva no Município estudado. Acredita-se que a atenção primária de saúde cumpre com seu papel na medida em que representa a porta de entrada para o sistema de saúde. A organização e gestão dos serviços perpassam pela suspeição e detecção de doenças, entre estas o câncer de mama. $O$ fato de que a primeira suspeita ou o diagnóstico tenha sido realizado na atenção primária demonstra que este nível de atenção mantém ações capazes de suprir essa necessidade e realizar os devidos encaminhamentos aos setores competentes.

A rede pública de referência para o rastreamento, detecção e tratamento do câncer de mama vem desenvolvendo seu papel no Município, pois apenas $17 \%$ dos casos teve a rede privada como porta de entrada. Porém, em outros municípios o estudo demonstra que o serviço privado desempenha papel mais relevante ao suprimir as lacunas do setor público, visto que $51,9 \%$ das buscas por serviços de saúde ocorreram primeiramente nas clínicas privadas ${ }^{10}$.

Embora 17\% dos prontuários das mulheres tenham evidenciado suspeita ou diagnóstico de câncer no setor privado, todas realizaram tratamento e algumas mantém seguimento na esfera pública em nível terciário, pois o Hospital Universitário de Santa Maria (HUSM) constitui-se em serviço de referência para a alta complexidade no Município, ou no nível secundário, serviço de mastologia municipal, já que os prontuários registram a assistência para estas mulheres. Não há como estimar o número exato de mulheres que foram diagnosticadas, realizaram tratamento e mantém seguimento na esfera privada, pois não há registro destes dados em um banco de dados único e na maioria das vezes não são notificados.

Presume-se, assim, que o Município estudado atende aos preceitos da organização do sistema de saúde vigente na medida em que a atenção à saúde da mulher é executada nos diferentes níveis de atenção à saúde (da básica à alta complexidade).

O câncer de mama é relativamente raro antes dos 35 anos, sendo que sua incidência cresce rápida e progressivamente após essa idade, sendo descoberto, principalmente, entre 40 e 60 anos ${ }^{11}$. Isso vem ao encontro dos dados identificados nesta pesquisa, em que também foi encontrada a idade de diagnóstico do câncer de mama na faixa etária entre 4160 anos, representando $57,03 \%$ da amostra. Vale destacar que $8,51 \%$ das mulheres estavam com menos de 40 anos quando confirmaram o diagnóstico e quatro mulheres tinham menos de 30 anos. Identificou-se ainda que maior número de diagnósticos de câncer de mama foi nas mulheres que tinham entre 41 e 50 anos, porém a prevalência até os 70 anos se sobressai, o que permite inferir que quanto mais avança a idade maior o risco de desenvolvimento da doença.

De acordo com a literatura quanto mais jovem a mulher apresentar neoplasia mamária, pior será o seu prognóstico ${ }^{12,13}$, sendo esse justificado pelo perfil molecular mais agressivo $0^{14}$ ou ainda, pela demora na realização do diagnóstico, visto que não são realizados rastreamentos mamográficos nessa parcela da população e o índice de suspeição clínica é baixo ${ }^{15,16}$.

Neste estudo, 91,7\% das mulheres eram da cor branca e 59,7\% eram casadas. Estes dados corroboram com os achados de outros autores ao observarem em seus estudos que a maioria das entrevistadas se autoreferiu como branca e casada ${ }^{17}$., porém este fator não foi considerado relevante em relação ao câncer de mama ${ }^{17}$.

A média de idade do diagnóstico foi de 56,1 anos, dado semelhante ao de outros estudos que avaliaram mulheres diagnosticadas com câncer de mama ${ }^{17,18}$. A partir dos 50 anos aumenta o número de casos de câncer de mama. Este fato possivelmente está relacionado com o processo de envelhecimento e ao tempo de exposiçãoaoestrogênio ${ }^{19,20}$.

Quanto aos fatores reprodutivos relacionados ao surgimento do câncer de mama, este estudo identificou que a menarca ocorreu, em média aos 13,1 anos. Este achado revela que estas mulheres podem não ter tido a menarca precoce como fator de risco para o desenvolvimento da doença, pois pesquisas indicam que a menarca precoce é aquela que ocorre anterior aos 12 anos de idade ${ }^{1,11}$.

Da mesma maneira, a menopausa tardia vem sendo relacionada com a doença, quando ocorre aos 55 anos ou mais, porém, nessa investigação a média de idade para a menopausa foi de 47,6 anos. Cabe ainda ressaltar que não é unanimidade considerar a menopausa tardia como um fator de risco ${ }^{21}$.

A indicação de tratamento cirúrgico mais radical geralmente está associada diversos fatores. A partir da análise dos dados desta pesquisa, na qual, os achados mamográficos, na maioria dos casos, foram o Birads4 (25\%) e o Birads5 (46\%), e que $66 \%$ das mulheres foram submetidas a mastectomia radical, podemos considerar que algum dos fatores como achados mamográficos suspeitos, diagnósticos tardios, tumores em estadiamento avançado, com maiores dimensões e presença de linfonodos comprometidos, estavam presentes no momento de tomada de decisão em relação ao tipo de cirurgia ${ }^{22}$. Em outro estudo que avaliou a sobrevida de mulheres com câncer de mama, a maioria delas realizou mastectomia, sendo que $60 \%$ dos casos apresentavam estadiamento clínico e tamanho do tumor avançados ${ }^{23}$. 
Atualmente, o tratamento para o câncer de mama tem apresentado transformações e aperfeiçoamentos condizentes com os avanços da medicina. As técnicas cirúrgicas, associada à radioterapia, à quimioterapia e hormonioterapia, têm como objetivo erradicar a doença local, reduzir ou prevenir a recorrência e prolongar a sobrevida ${ }^{24}$.

Há uma grande prevalência de receptores hormonais positivos nos tumores de mama em geral, fato que pode justificar o grande percentual de mulheres desse estudo em tratamento com hormonioterapia $(41 \%)^{24}$. Além disso, a utilização de terapia hormonal está associada à maior sobrevida livre de doença e, possivelmente, à sobrevida global, além de reduzir a incidência do câncer de mama contralateral e diminuir a ocorrência de micrometástase ${ }^{25}$.

\section{Considerações Finais}

Conclui-se que, dos prontuários analisados, o nível de atenção primária do SUS foi o local de maior probabilidade como serviço de primeira suspeita do câncer de mama em mulheres no município de Santa Maria. A média de idade de 56,1anos, a cor branca auto declarada e o estado civil casada foram as características sociodemográficas encontradas. Quanto as características reprodutivas, a idade média para menarca precoce, para menopausa tardia, assim como,primeira gestação não foram consideradas fatores de risco para o desenvolvimento da doença conforme retrata a literatura.

Os achados mamográficos foram suspeitos para o câncer de mama, sendo que a mastectomia radical tem sido a técnica de escolha seguida da hormonioterapia como terapêutica adjuvante mais recorrente.

Cabe ressaltar a relevância dessa pesquisa, na medida em que contribui para a identificação das características de mulheres com câncer de mama em Santa Maria, pois essas informações possibilitam o reconhecimento de elementos peculiares da população assistida no Município. Estes dados constituem-se em subsídios para a elaboração de estratégias de detecção precoce do câncer de mama principalmente em grupos populacionais com as características apresentadas no estudo. Isso torna possivvel, intervenções efetivas que podem prevenir a necessidade de tratamentos mais invasivos e mutiladores.

As limitações do estudo estão na falta de informações nos prontuários não sendo possível identificar dados relevantes para a temática como aleitamento materno, paridade, histórico familiar, entre outros. Ainda a ausência de informatização dos registros/prontuários em ambos os serviços investigados dificultou o acesso aos mesmos.

\section{Referências}

1. Silva PA, Riul SS. Câncer de mama: fatores de risco e detecção precoce. Rev. bras. enferm. 2011;64(6):1016-21.

2. Brasil. Ministério da Saúde. Instituto Nacional de Câncer José Alencar Gomes da Silva. Coordenação de Prevenção e Vigilância Estimativa 2014: Incidência de Câncer no Brasil. Instituto Nacional de Câncer José Alencar Gomes da Silva. Coordenação de Prevenção e Vigilância. Rio de Janeiro: INCA, p. 1-124, 2014.

3. Dutra MC, Rezende MA, Andrade VP, Soares FA, Ribeiro MV, Paula EC, et al. Imunofenótipo e evolução de câncer de mama: comparação entre mulheres muito jovens e mulheres na pós-menopausa. Rev. BrasGinecol. Obstet. 2009;31(2):54-60.

4. Brasil. Ministério da Saúde. Instituto Nacional de Câncer José Alencar Gomes da Silva. Programa Nacional de Controle do Câncer de Mama. Versão revista e ampliada do Programa Viva Mulher, desmembrado em Programa Nacional de Controle do Câncer do Colo do Útero e Programa Nacional de Controle do Câncer de Mama (INCA, 2010), elaborado pela Divisão de Apoio à Rede de Atenção Oncológica. 2011.

5. Aragão JA, Reis FP, Marçal AC, Santos MRV, Cardoso EC, Xavier LMF, et al. Nível de estadiamento das mulheres submetidas a tratamento do câncer de mama. ScientiaPlena.2012;8(3):1-6.

6. HöfelmannDA, Anjos JC. Autoavaliação de Saúde e Câncer de Mama em Mulheres de Cidade do Sul do Brasil. Revista Brasileira de Cancerologia, 2012;58(2):209-22.

7. Brasil. Ministério da Saúde. Instituto Nacional de Câncer José Alencar Gomes da Silva. Coordenação Geral de Ações Estratégicas. Coordenação de Prevenção e Vigilância. Estimativa 2012: incidência de câncer no Brasil. Rio de Janeiro: Inca, 2011. 118p.

8. Brasil. Ministério da Saúde. Instituto Nacional de Câncer José Alencar Gomes da Silva. Ações e Programas. Agenda Estratégica. 2011.[acessado 2013 ago 16] Disponível em: http://www2.inca.gov.br/wps/wcm/connect/acoes_programas/ site/home/agenda_estrategica/apresentacao_agenda_estrategica. 
9. Santos Junior JC, Soares LFM. Câncer de Mama. In: Vieira SC, Lustosa AML, Barbosa CNB, Teixeira JMR, De Brito LXE, Soares LFM, e col. Oncologia Básica. 1ed. Teresina, PI: Fundação Quixote; 2012. p. 41-60.

10. Rezende MCR, Koch HA, Figueiredo JA, Thuler LCS. Causas do retardo na confirmação diagnóstica de lesões mamárias em mulheres atendidas em um centro de referência do Sistema Único de Saúde no Rio de Janeiro. Rev. Bras. Ginecol. Obstet. 2009;31(2):75-81.

11. Inca. Instituto Nacional de Câncer (Brasil). Ações de enfermagem para o controle do câncer: uma proposta de integração ensino-serviço. 3ed.atual.amp. Rio de Janeiro: INCA, 2008.

12. Brasil. Ministério da Saúde. Secretaria de Assistência à Saúde. Instituto Nacional de Câncer. Câncer no Brasil: dados dos registros de base populacional. Rio de Janeiro: INCA, v. 4, 2010.

13. Rudat V, El-Sweilmeen H, Fadel E, Brune-Erber I, Ahmad Nour A, Bushnag Z, et al. Age of 40 years or younger is an independent risk factor for locoregionalfailure in early breast cancer: a single-institutional analysis in Saudi Arabia. J Oncol. 2012;(2012):1-10.

14. Castiglione M, Aebi S. The enigma of young age. Annoncol. 2006;17(10):1475-7.

15. Kothari AS, Fentiman IS. Breast cancer in youngwomen. Int J ClinPract. 2002; 56(3):184-7.

16. Crippa CG, Hallal ALC, Dellagiustina AR, Traebert EE, Gondin G, Pereira C. Perfil Clínico e Epidemiológico do Câncer de Mama em Mulheres Jovens. ArqCatarin Med.2003;32(3):50-58.

17. Vieira EM, Yoshinari Júnior GH, Souza HCC, Mancini MPM, Perdoná GSC. História reprodutiva e sexual de mulheres tratadas de câncer de mama. RevBrasGinecol Obstet. 2013;35(2):78-83.

18. Trufelli DC, Miranda VC, Dos Santos MBB, Fraile NMP, Pecoroni PG, Gonzaga SFR, et al. Análise do atraso no diagnóstico e tratamento do câncer de mama em um hospital público. Ver AssocMed Bras. 2008;54(1):72-76.

19. Pinho VFS, Coutinho ESF. Variáveis associadas ao câncer de mama em usuárias de unidades básicas de saúde. Cad. Saúde Pública. 2007;23(5):1061-69.

20. Gonçalves LLC, Lima AV, Brito ES, Oliveira MM, Oliveira LAR, Abud ACF, et al. Fatores de risco para câncer de mama em mulheres assistidas em ambulatório de oncologia. Rev. de Enfermagem. 2010;18(3):468-72.

21. Matos JC, Pelloso SM, Carvalho MDB. Prevalência de fatores de risco para o câncer de mama no município de Maringá, Paraná.Rev. Latino-Am. Enfermagem.2010;18(3):1-8.

22. Gonçalves LLC, Santos SB, Marinho EC, Almeida AM, Santos AHS, Barros AMMS, et al. Câncer de mama feminino: aspectos clínicos e patológicos dos casos cadastrados de 2005 a 2008 num serviço público de oncologia de Sergipe. Rev. BrasSaude Mater. Infant.2012;12(1):47-54.

23. Moraes AB, Zanini RR, Turchiello MS, Riboldi J, Medeiros LRF. Estudo da sobrevida de pacientes com câncer de mama atendidas no hospital da Universidade Federal de Santa Maria, Rio Grande do Sul, Brasil. Cad. Saúde Pública.2006;22(10):2219-28.

24. Brito C, Portela MC, Vasconcellos MTL. Assistência oncológica pelo SUS a mulheres com câncer de mama no estado do Rio de Janeiro. Rev. Saúde Pública. 2005;39(6):874-81.

25. Leite FMC, Bubach S, Amorim MHC, Castro DS, Primo CC. Mulheres com diagnóstico de câncer de mama em tratamento com Tamoxifeno: perfil sociodemográfico e clínico. Revista Brasileira de Cancerologia. 2011;57(1):15-21. 


\section{Hedioneia Maria Foletto Pivetta}

Endereço para correspondência - Rua: Av. Roraima, $n^{\circ} 1000$, Centro de Ciências da Saúde, Bairro: Camobi, CEP: 97.105-900, Santa Maria, RS, Brasil.

E-mail: hedioneia@yahoo.com.br

Lattes: http://lattes.cnpq.br/9518521941876440

Adriana Cielo - adrianacielo025@hotmail.com

Marina Segala - marina_sgl@hotmail.com

Gustavo do Nascimento Petter - gustavo.petterr@hotmail.com

Thais Nogueira de Oliveira Martins - thais21_oliveira@hotmail.com

Betina Pivetta Vizzotto - be_vizzotto@hotmail.com

Elenir Terezinha Rizzetti Anversa - eleanversa@hotmail.com

Melissa Medeiros Braz - melissabraz@hotmail.com

Enviado em 07 de novembro de 2015. Aceito em 15 de agosto de 2016. 